\title{
Mittendrin statt nur dabei: Ethnographie als Methodologie in der Humangeographie
}

\author{
M. Müller \\ Universität St. Gallen, Varnbüelstrasse 19, St. Gallen, Switzerland \\ Correspondence to: M. Müller (martin@martin-muller.net)
}

\begin{abstract}
Zusammenfassung. Interest in the lived mundane practices and embodied experience of subjects has seen a tremendous upsurge in human geography in the past years. With its focus on social interaction and concern with subjects' lifeworlds, ethnography suggests itself as a suitable methodological approach to match this interest. Against the lack of a sustained debate in German-speaking human geography, this special issue seeks to illustrate the potential of ethnography for different conceptual approaches with the help of empirical examples. It is the task of this editorial to review key issues associated with ethnographic research. In so doing, it does not equate ethnography with the method of participant observation, but rather understands it as a methodology with specific implications for the responsibility and position of the researcher, the interpretation of the material and the construction of a narrative.
\end{abstract}

\section{Ethnographie - mittendrin statt nur dabei}

I like to inquire into everything. Hercule Poirot is a good dog. The good dog follows the scent, and if, regrettably, there is no scent to follow, he noses around. (Hercule Poirot in Agatha Christies Peril at End House)

Agatha Christies Beschreibung ihres Protagonisten Hercule Poirot ist nicht minder zutreffend als Allegorie für die Ethnographie. Der Ethnograph als Spürhund, als unauffälliger Detektivin und induktive Ermittlerin, interessiert an allen Kleinigkeiten, aus denen sich schliesslich grössere $\mathrm{Zu}$ sammenhänge erschliessen, ist sicher kein unpassendes Bild. Die Wahl von Christies Werk und Wirken als Leitmotiv für diesen Beitrag ist daher durchaus bewusst: Ethnographische Forschung zeichnet sich häufig durch dichte aber zugleich kurzweilige Beschreibungen von Alltagsinteraktionen aus und lässt damit, nicht unähnlich den Romanen von Christie, fremde Lebenswelten eigentümlich vertraut erstehen. Um diese dichten Beschreibungen überhaupt möglich zu machen, erfordert diese Art von Forschung die Teilhabe an den Lebenswelten der Forschungssubjekte. Die ethnographische Forscherin ist eben mittendrin und nicht nur dabei. Mit Harry Wolcott (1973) lernen wir die Welt von Ed Bell, Rektor der Taft-Grundschule, kennen, der sich mit widerspenstigen Schülerinnen und besorgten Eltern, kleinen Wehwehchen und großen Budgetkürzungen herumschlagen muss. Wir begeben uns mit Laud Humphreys (1970) ver- deckt auf die Suche nach homosexuellen Begegnungen auf öffentlichen Toiletten am Rande der Legalität und folgen mit Julian Orr (1996) wie Büromaschinentechniker sich und ihre Arbeit entwerfen. Mittendrin statt nur dabei versuchen wir sonst unbekannte soziale Welten zu begreifen - die Ausbildung von Ärzten (Becker et al., 1961), das Schmuggeln und Dealen von Drogen (Adler, 1985) oder das feucht-modrige Reich der Pilzsammler (Fine, 1998).

Für die Geographie erscheint die Ethnographie vor allem wegen ihrer Beschäftigung mit sozialen Praktiken und gelebter Erfahrung relevant, die in den vergangenen Jahren zunehmend in den Fokus der Disziplin gerückt ist. Das Interesse am „doing“ und „,making“ von Geographien, an Performativität, Machttechniken, gelebter Praxis und verkörperten Subjekten, kennzeichnet sowohl feministische Geographien (z.B. Schurr und Segebart, 2013; Wastl-Walter, 2010) als auch die neuere Kulturgeographie (z.B. Everts et al., 2011; Strüver, 2011), ebenso die Wirtschaftsgeographie (z.B. Boeckler und Berndt, 2008), Stadtgeographie (z.B. Dirksmeier und Helbrecht, 2010) und Politische Geographie (z.B. Füller und Marquardt, 2009; Müller, 2008). Auch die Erfassung von Materialität, die bei Arbeiten im Anschluss an die Phänomenologie, Non-Representational Theory oder Akteur-Netzwerk-Theorie eine wichtige Rolle spielt, ist mit ethnographischer Forschung oft besser möglich als mit anderen Zugängen. Dabei bedeutet eine Hinwendung zur Ethnographie keineswegs eine Abkehr von stärker textorientierten Methodologien. Im Gegenteil, die Ordnung des Diskurses und die Unordnung der Praxis (Korf, 2004) 
sind zwei Seiten einer Medaille - je nach Fragestellung und Erkenntnisinteresse können sie unterschiedliche Antworten geben.

Die Zeit scheint also reif für mehr Ethnographie in der Humangeographie und tatsächlich ist in neuerer Zeit im deutschsprachigen Raum eine zunehmende Zahl an Arbeiten zu verzeichnen, die in wesentlichen Teilen ethnographischen Ansätzen folgen (z.B. Berndt, 2004; Everts, 2008; Lange, 2007; Müller, 2009; Rothfuß, 2012 unter den neueren Monographien). Auch im englischsprachigen Raum hat die Ethnographie, nach Klagen über ihr Schattendasein (Herbert, 2000; Megoran, 2006), zuletzt in der Humangeographie an Popularität gewonnen, was sich auch in entsprechenden Lehr- und Handbüchern widerspiegelt (z.B. Crang und Cook, 2007; Watson und Till, 2010). Vor diesem Hintergrund soll das vorliegende Themenheft dazu dienen, eine systematische Diskussion über die Ethnographie anzustossen und zu erörtern, welche Rolle und Bedeutung sie in geographischen Forschungsarbeiten haben könnte und sollte.

Anders als in den meisten deutschsprachigen Methodenlehrbüchern der Geographie, die stets von teilnehmender Beobachtung sprechen, soll mit dem Begriff Ethnographie hier bewusst eine Methodologie verstanden werden. Während die Methode der teilnehmenden Beobachtung dafür grosse $\mathrm{Be}$ deutung besitzt, ist Ethnographie nicht einfach ein anderes Verfahren zum Sammeln von Daten, sondern vielmehr ,a way of seeing“, wie es Wolcott (1999) formuliert. „Theory, writing, and ethnography are inseparable material practices“, schreibt Denzin (1997:xii) und bringt damit zum Ausdruck, dass Ethnographien immer theoretisch rückgebunden werden und über den Prozess ihrer eigenen Produktion reflektieren müssen. Dieser Charakter der Methodologie soll in den folgenden Abschnitten in dreierlei Hinsicht dargestellt werden: Zum Ersten bedingt das Forschungsinteresse am Alltagsleben der Forschungssubjekte eine besondere Position und Verantwortlichkeit der Forscherin. Zum Zweiten müssen der Status und die Interpretation des Materials reflektiert werden. Zum Dritten schliesslich ist der Prozess des Schreibens und der Konstruktion eines Narrativs von zentraler Bedeutung.

\section{Alltagspraxis und Verantwortlichkeit}

Try and vary your methods as you will, your tastes, your habits, your attitude of mind, and your soul is revealed by your actions. (Agatha Christies The ABC Murders)

Ethnographie konzentriert sich primär auf die Erfassung und Interpretation von sozialen Praktiken und gelebter Erfahrung, nicht auf sprachliche Repräsentationen. Solche Repräsentationen mögen Teil von sozialen Praktiken und gelebter Erfahrung sein, aber sie sind nicht notwendigerweise gleichbedeutend mit ihnen (Agar, 1996). Ganz im Gegenteil, handeln oder fühlen Subjekte doch oft im Sinne von „Talk
Left, Walk Right" anders als sie es behaupten zu tun. In der Ethnographie geht es darum, soziale Prozesse, gelebte Erfahrung und Bedeutungszuschreibungen induktiv zu erfassen, ohne ihnen sofort mit vorgefertigten theoretischen Konzepten zu begegnen. Das (utopische) Idealbild der Ethnographin wird daher häufig als „tabula rasa“ beschrieben: um sich an die Perspektive der Forschungssubjekte anzunähern, müssen theoretische Überzeugungen und Vorwissen über Bord geworfen oder zumindest hinterfragt werden. Anstatt mit Hypothesen oder theoretisch abgeleiteten Fragestellungen $\mathrm{zu}$ operieren, versucht die Ethnographin das Besondere aus dem Alltäglichen heraus zu verstehen: ,in order to understand the spectacular event, it is necessary to see it in relation to the everyday pattern of life“" (Whyte, 1943:xvi).

In der Ethnographie können dabei prinzipiell unterschiedliche methodische Zugänge zum Einsatz kommen. Zwar gilt die teilnehmende Beobachtung als das methodische Herzstück, jedoch finden auch Interviews, Film- und Tonaufnahmen, Dokumente oder quantitative Erhebungen ihren Platz. Wenn daher im Folgenden Beispiele aus der teilnehmenden Beobachtung gewählt werden, so deshalb, weil diese Methode der Forscherin in besonderer Weise erlaubt, in das Alltagsgeschehen von Subjekten eingebunden zu sein. Teilnehmendes Beobachten erscheint dabei häufig als Paradox. Beobachten impliziert eine gewisse Distanz zum Geschehen, Teilnehmen hingegen eine unmittelbare Nähe. Genau in diesem Spannungsfeld bewegt sich auch die Ethnographin, ob mit der teilnehmenden Beobachtung oder mit anderen methodischen Instrumenten (vgl. Adler et al., 1986; Fine, 1994). Allzu grosse Nähe birgt die Gefahr des ,going native": die Perspektive der Forschungssubjekte wird zumindest teilweise übernommen, so dass diese nicht mehr kritisch reflektiert werden kann. Allzu grosse Distanz hingegen riskiert die Akzeptanz als ein Mitglied der Gruppe und gefährdet damit die Herausbildung eines adäquaten Verständnisses sozialer Kontexte.

Mit dem Forschungsinteresse am Alltagsleben von Personen ergeben sich gleichzeitig auch eine Reihe moralischer und ethischer Dilemmata (z.B. Fine, 1994; Korf, 2004). Wie in dem Zitat von Agatha Christie oben angedeutet, stellen viele ethnographische Zugänge eine gewisse Intimität her und Forschungssubjekte haben häufig keine direkte Kontrolle mehr, welche Informationen sie an die Ethnographin weitergeben. Daraus erwachsen einige zentrale ethische Fragen: Bis zu welchem Grad mache ich meine wissenschaftlichen Intentionen transparent (Herrera, 2003)? Wie repräsentiere ich meine Forschungssubjekte angemessen? Inwieweit nutze ich freundschaftliche oder intime Beziehungen zum Sammeln von Daten? Wie vermeide ich Schaden für meine Forschungssubjekte, der durch meine Forschung entstehen könnte? Besitze ich eine moralische Verpflichtung, in meiner Feldforschung Unrecht zu verhindern oder umgekehrt, Gruppen in ihrem Kampf um Recht zu unterstützen? Ethnographie ist damit mehr als unpolitisches Datensammeln: sie bedeutet, aktiv Verantwortung zu übernehmen. 
3 Fakt...

Exactly! It is absurd - improbable - it cannot be. So I myself have said. And yet, my friend, there it is! One cannot escape from the facts. (Hercule Poirot in Agatha Christies Murder on the Orient Express)

Was unterscheidet Agatha Christie und die Wissenschaft, was Roman und Ethnographie? Man ist zunächst verführt die Faktizität als zentrales Kriterium heranzuziehen: In der Ethnographie geht es um Fakten, in Romanen um Fiktion. Tatsächlich verstehen sich traditionelle Ethnographien als realistische Beschreibungen einer bestimmten Kultur, sei es einer Ethnie, einer Organisation oder einer anderen Art von Gemeinschaft. van Maanen (1988:45-72) führt vier zentrale Merkmale solcher realistischen Ethnographien auf.

1. Autorität durch Erfahrung beschreibt den Anspruch auf Authentizität, gewonnen zum Beispiel durch eine lange Verweildauer im Feld oder durch intensive Auseinandersetzung mit einer bestimmten sozialen Gruppe. Beschreibungen einer sozialen Gruppe werden als objektiv und unabhängig von der Beobachterin angesehen; die Autorin und ihre Subjektivität werden ausgeblendet.

2. Minutiöse Beschreibung von Interaktionen ist die typische Form realistischer Ethnographien. Die Präsentation von Details dient meist der Darstellung von typischen Situationen und untermauert somit ein bestimmtes Argument.

3. Das Einnehmen einer emischen Perspektive ergibt sich aus den ersten beiden Merkmalen. Die Autorin gibt nicht ihre eigenen Eindrücke, sondern die Perspektive der Forschungssubjekte wieder, indem Interaktionen ,straight from the horse's mouth“ (van Maanen, 1988:49) transkribiert werden.

4. Realistische Ethnographien beanspruchen zumindest implizit interpretative Omnipotenz. Die Autorin hat das letzte Wort in der Interpretation der dargestellten Phänomene, alternative Interpretationen werden oft nicht diskutiert. Häufig werden Beobachtungen aus der Feldforschung verwendet, um bestimmte Theorien zu illustrieren oder zu modifizieren.

\section{...oder Fiktion?}

I have always been so sure - too sure... But now I am very humble and I say like a little child: "I do not know..." (Hercule Poirot in Agatha Christies Curtain: Poirot's Last Case)

Mit der poststrukturalistischen „Krise der Repräsentation“ (Clifford und Marcus, 1986) verlor das Kriterium der Faktizität an Validität. Anders als realistische Ethnographien erheben poststrukturalistische Ethnographien keinen Anspruch auf Wahrheit und Objektivität, sondern erkennen an, dass wissenschaftliche Erkenntnis immer partiell und situiert ist. Sprache - und nicht-sprachlichen Medien wie Film (Trinh, 1991, siehe auch Schurr in diesem Heft) - kommt daher zumindest in einer poststrukturalistisch orientierten Ethnographie eine andere Rolle zu als in vielen anderen Formen wissenschaftlicher Prosa. Es geht nicht mehr um die akkurate Repräsentation einer vermeintlich objektiv zugänglichen Wahrheit, sondern vielmehr um die Erschaffung eines plausiblen Narrativs, einer plausiblen Deutung (Denzin, 1997:10-13).

Realität wird durch ethnographisches Erzählen neu erschaffen. Ethnographie ist somit im besten Sinne Fiktion in Rückgriff auf die lateinische Wortwurzel fingere, die soviel bedeutet wie ,gestalten“ oder „erschaffen“. Von der poststrukturalistischen Ethnographin wird genau dasselbe gefordert wie von der Romanautorin: die Erschaffung einer überzeugenden Erzählung. So beschrieb auch Mauss (1947:8) bereits vor mehr als 60 Jahren die Rolle des Ethnographen: ,à la fois chartiste, historien, statisticien et aussi romancier capable d'évoquer la vie d'une société tout entière." (siehe hierzu auch explizit Geertz, 1988:140; Law, 2004; Visweswaran, 1994). Doch während im Kriminalroman Agatha Christies die Spannung zwischen Plausibilität und Realität schliesslich aufgelöst wird, indem der wahre Mörder enttarnt wird, kann die Ethnographie nicht mit einer solchen Verankerung in einer objektiven Realität aufwarten. „Ethnographic descriptions are not privileged, just particular: another country heard from... [They are not] about discovering the Continent of Meaning and mapping out its bodiless landscape" (Geertz, 1973:23, 20). Macht diese Tatsache ethnographische Forschung beliebig? Keineswegs. Ethnographie ist zwar eine Erzählung unter vielen anderen, die aber mit dem Wissen um ihre Kontingenz über die Bedingungen ihrer Produktion reflektieren kann (Hammersley und Atkinson, 1995; Marcus, 1994:16-22). Über Reflexion kann in der Ethnographie also - anders als im Roman - die Partialität und Situiertheit von jeglicher Wissensproduktion offengelegt werden (Katz, 1992). Damit gelangen wir zwar nicht näher an eine Wahrheit, aber erhalten quasi die interpretatorischen Baupläne des ethnographischen Narrativs (siehe auch Frers in diesem Heft).

Eine solche Reflexion erfordert auch die Thematisierung der Position und Rolle der Autorin in der Erschaffung von Ethnographien - ein zentrales Anliegen gerade feministischer Forschung. Der ,god-trick of seeing everything from nowhere" (Haraway, 1991:189), der realistische Ethnographien kennzeichnet, wird als gescheitert angesehen. Interessanterweise wählt die poststrukturalistische Ethnographie hier einen anderen Weg als die Diskursanalyse, um mit dem Problem der Relativität von Wissen über die Welt umzugehen. Während einige Stränge der Diskursanalyse durch lexikometrische Methoden versuchen, die Autorin zunächst soweit wie möglich aus dem Text zu subtrahieren, tritt die Ethnographie quasi die Flucht nach vorne an und 
bemüht sich, die Position der Autorin möglichst transparent zu machen. In einer Ethnographie als Methodologie geht es deshalb darum, Wissen zu situieren, die eigene Positionalität zu reflektieren und die Autorität durch Erfahrung realistischer Ethnographien in Frage zu stellen (Madison, 2005; Rose, 1997).

Ein solcher Schritt reformuliert auch das Verhältnis von Theorie und Empirie. Ethnographische Empirie diktiert der Forscherin nicht, gleichsam wie in einem naiven Empirismus, die Erkenntnisse in die Feder. Dieses theorielose Beobachten als zentrale Methode der Geographie geriet bereits mit dem Kieler Geographentag 1969 stark in Kritik: „Die Theorie ist also vor der Beobachtung. Fragen Sie nicht, was vor der ersten Theorie war: den theorielosen Zustand gab es nie; wer beobachtet, hat immer schon etwas gelernt. ... Beobachtungen sind prinzipiell nicht fundamentaler und nicht sicherer als Theorien“" (Bartels und Hard, 1975:169, Hervorhebungen im Original). Gleichzeitig aber kann es auch nicht darum gehen mit der Theoriebrille gerüstet ,abstrahierte Entitäten zu einheitlichen Mustern zusammenzufügen“" (Geertz, 1983 [1973]:26, zitiert im Beitrag von Verne). Material und Theorie müssen vielmehr in einen Dialog treten; Theorien auf ihre Eignung ein konkretes empirisches Phänomen zu beschreiben überprüft und schliesslich revidiert werden. Die Offenheit gegenüber dem Unerwarteten und die Erfassung der mikrosozialen Aushandlung von Bedeutung sind dabei die zentralen Merkmale ethnographischer Forschung.

\section{Ethnographie schreiben}

[T]he more personal you are the better! This is a story of human beings - not dummies! ... Write the thing your own way. (Dr. Reilly in Agatha Christies Murder in Mesopotamia)

Für Agatha Christie waren Notizbücher ähnlich zentral als Zwischenlager von Ideen und Beobachtungen wie sie es wohl für die mit teilnehmender Beobachtung arbeitende Ethnographin sind (Sanjek, 1990). Christies Notizbücher bestehen aus einem ungeordneten Sammelsurium an Einträgen. Entwürfe für Charaktere und Beobachtungen aus dem eigenen Alltag stehen neben Einkaufslisten und Termineinträgen. Diese Notizen mussten so geordnet werden, dass aus dem scheinbaren Chaos zusammenhängende Handlungsverläufe entstanden. Dabei wusste Christie manchmal selbst nicht, wer der Mörder sein würde, als sie begann ein Buch zu schreiben (Curran, 2009).

Auch die Ethnographin sieht sich nach Abschluss der Feldforschung mit der Aufgabe konfrontiert eine Fülle von Einzelnotizen in Narrative zu giessen und gegen verschiedene Theorien zu lesen. Für Geertz (1973:19) besteht die Tätigkeit der Ethnographin zuvorderst aus dem Schreiben. Damit grenzt er sich von traditionellen Definitionen von Ethnographie als Beobachtung, Dokumentation und Analyse ab. Geertz hat für ethnographisches Schreiben den Begriff „dich- te Beschreibung“ geprägt. Im Unterschied zu „dünnen Beschreibungen“" werden darin nicht nur soziale Handlungen, sondern auch deren (mögliche) Bedeutungen beschrieben. Dichte Beschreibung ist also Beschreibung und Interpretation zugleich - Clifford Geertz spricht von Einschreibung (inscription) - und erkennt somit an, dass es keine Daten in roher Form gibt. Sie bereitet Beobachtungen für die Leserin auf und macht eine gewisse Kultur, eine soziale Gruppe lesbar.

Kernmerkmal der dichten Beschreibung ist die detaillierte Wiedergabe von sozialen Interaktionen. Indem wir dieses Material den Lesern und Leserinnen zur Verfügung stellen, ermöglichen wir ihnen, ähnlich wie bei Agatha Christie, selbst an der Ermittlung teilzunehmen und auf Basis der Beschreibungen von Situationen eigene Interpretationen zu bilden. Die Darstellung des empirischen Materials ermöglicht somit einen gewissen Grad an interpretatorischer Transparenz. Dabei ist allerdings die Beschreibung von sozialen Interaktionen keineswegs als eine authentische Erfassung zu verstehen, sondern vielmehr als eine Rekonstruktion, wie Fine (1994:277-278) feststellt:

We become playwrights, reconstructing a scene for the insight of our readers ... Notetaking and writing demand transformation and recontextualization. We claim that the scene really happened, but the scene did not happen in precisely the form we announce. We are like those popular biographers who, in order to make a scene compelling and "real", create dialogue that is "likely" to occur and that, in the process, supports our own arguments and morals.

Ethnographien sind deshalb - im Sinne des Zitats von Dr. Reilly - immer persönliche Geschichten: verschiedene Ethnographinnen würden dieselbe Szene anders beschreiben und anders interpretieren. Umso wichtiger ist es, Transparenz zum Forschungsprozess, der eigenen Positionalität und den theoretischen Annahmen zu schaffen.

\section{Die Beiträge im Heft}

Die Beiträge in diesem Themenheft versuchen exemplarisch, das Potenzial der Ethnographie für neuere Ansätze in der Humangeographie zu heben. Sie setzen sich mit Phänomenologie (Lars Frers), Netzwerken und Hermeneutik (Julia Verne), Vermarktlichung (Stefan Ouma) und Performativität (Carolin Schurr) auseinander. Man könnte sie im klassischen Schema der Humangeographie ordnen und dabei zwei in der Kultur- und Sozialgeographie (Frers, Verne), einen in der Wirtschaftsgeographie und einen in der Politischen Geographie (Schurr) verorten. Das aber würde die zahlreichen methodologischen und methodischen Gemeinsamkeiten verdecken, die sich aus dem ethnographischen Zugang ergeben und die von allen geteilte Beschäftigung mit gelebten sozialen Interaktionen. 
Für Lars Frers ist ethnographisches Forschen leibliches Erfahren mit all seinen sinnlichen Implikationen und er demonstriert dies am Beispiel seiner Forschung an einem Aussichtspunkt an der norwegischen Touristenroute. Er reflektiert den ethnographischen Forschungsprozess als ein Changieren zwischen Nähe und Distanz, Gemenge und Analyse, Gegenwart und Aufzeichnung. Mittendrin statt nur dabei - Lars Frers nimmt das Motto eines deutschen Sportsenders, auf dem es regelmässig schwitzende Körper, Tränen der Enttäuschung, Jubelschreie, überkochende Aggressionen zu sehen gibt, wörtlich.

Julia Verne betreibt Ethnographie im Geiste Clifford Geertz' als Hermeneutik. Für sie bedeutet ethnographische Forschung, ähnlich wie für Lars Frers, Offenheit gegenüber Überraschungen, die aus der Empirie resultieren und erlauben, Prozesse der Emergenz nachzuzeichnen. Theorien versteht sie dabei als eine „Beirrung“ - als vorläufige Erkenntnisheuristiken, die es zu hinterfragen und zu revidieren gilt. Für Julia Verne ist das klassische Netzwerkverständnis eine solche Beirrung, die sie mit der gelebten Migrationserfahrung einer sansibarischen Familie konfrontiert.

Bei Stefan Ouma dient die ethnographische Forschung dazu, einen Blick hinter oft selbstverständlich scheinende soziale Erscheinungen zu werfen. Für ihn ist eine solche der Markt, den er in der Tradition einer durch Michel Callon beeinflussten Wirtschaftssoziologie als sozio-materiellen Kalkulationsmechanismus versteht. Diese Kalkulation zeichnet sich durch hohe Kontingenz aus und bedarf deshalb einer stetigen Stabilisierung. Stefan Ouma legt dar, welchen Beitrag ein ethnographischer Ansatz an ein solches Verständnis von Märkten und ihrer Performativität leisten kann und bezieht sich dabei auf seine Forschung zur Integration von ghanaischen Kleinbauern in globale Agrarmärkte.

Im letzten Beitrag schliesslich sieht Carolin Schurr Ethnographie als eine Möglichkeit an Performanzen teilzunehmen und methodisch das Versprechen einzulösen, das mit der Hinwendung zum Konzept der Performativität entsteht. Sie konzentriert sich dabei auf eine Spielart der Ethnographie, die visuelle Ethnographie. Carolin Schurr präsentiert uns Videoaufzeichnungen von ecuadorianischen Politikerinnen im Wahlkampf und fragt danach, wie in Auftritten und Selbstpräsentationen bestimmte Identitäten reproduziert oder in Frage gestellt werden. Dabei reflektiert sie sich auch die Grenzen dieser Herangehensweise und plädiert für eine Ergänzung durch andere Ansätze.

Aus dem Querschnitt dieser Beiträge zeichnet sich ein Bild der Ethnographie in der Humangeographie, das sich durchaus vom vorherrschenden Verständnis in der Anthropologie und Soziologie unterscheidet. Die Mehrzahl der Beiträge ist nicht primär am holistischen Verständnis einer sozialen Gruppe oder Kultur interessiert, sondern an einem bestimmten Phänomen, sei es nun der Konstruktion von Märkten, Migrationsnetzwerken oder politischen Performanzen. Der Zweck von ethnographischer Forschung ist also von Beginn an deutlich enger abgesteckt. Dabei tritt auch die detailreiche, minutiöse Wiedergabe von Interaktionen hinter einer stärker zweckorientierten, summierenden Beschreibung zurück. Die Beiträge sind auch überwiegend traditionell strukturiert - Theorie, Methode, Empirie - anstatt die Forschung zum Beispiel von einem empirischen Narrativ her aufzurollen. Ausführlichen Reflexionen über den ethnographischen Forschungsprozess und die Interpretation des Materials sind aufgrund der Länge von Zeitschriftenartikeln Grenzen gesetzt. Die Monographie ist hier zweifellos die besser geeignete Präsentationsform.

Die Beiträge zeigen auch, dass eine Ethnographie in der Humangeographie Strategien entwickeln muss um einer Welt gerecht zu werden, die sich durch globale räumliche Verflechtungen auszeichnet. Das kann im Sinne einer multilokalen Ethnographie wie bei Julia Verne geschehen, die den Reisewegen von Menschen und Objekten folgt (siehe Falzon, 2009; Peck und Theodore, 2010). Eine solche Strategie des „follow the people“ erfordert auch von der Forscherin hohe Mobilität und die Fähigkeit sich auf unterschiedliche Kontexte einzustellen. Es kann aber auch im Projekt der globalen Ethnographie passieren, das sich - ähnlich wie bei Stefan Ouma - dafür interessiert, wie globale Prozesse sich in Lokalitäten niederschlagen (Gille und Ó Riain, 2002).

Für die Humangeographie bedeutet der Versuch an der Ethnographie einen Lernprozess, im Verlauf dessen sich eigene Varianten und Spielarten geographischer Ethnographie herausbilden können und sollen. Ein ethnographisches Narrativ zu weben ist keine einfache Aufgabe. Es dauert Jahre, manchmal Jahrzehnte, um genügend aussagekräftiges Material für eine dichte Beschreibung zu sammeln. Aus zwei Monaten Feldforschung wird manchmal eine Seite Text oder auch keine. Ethnographie benötigt gerade aufgrund der schwierigen Planbarkeit der Forschung Freiräume jenseits der zunehmend schnelllebigen Verwertungszyklen in der Wissenschaft. Geben wir ihr diese - nicht zuletzt für eine reichere, buntere, spannendere Geographie!

Believe me, nurse, the difficulty of beginning will be nothing to the difficulty of knowing how to stop. At least that's the way it is with me when I have to make a speech. Someone's got to catch hold of my coat-tails and pull me down by main force. (Dr. Reilly in Agatha Christies Murder in Mesopotamia)

\section{Literatur}

Adler, P. A.: Wheeling and dealing: an ethnography of an upperlevel drug dealing and smuggling community, New York, Columbia University Press, 1985.

Adler, P. A., Adler, P., und Rochford,E. B.: The politics of participation in field research, Urban Life, 14, 363-376, 1986.

Agar, M.: The professional stranger: an informal introduction to ethnography, 2nd Edn., London, Academic Press, 1996. 
Bartels, D. und Hard, G.: Lotsenbuch für das Studium der Geographie als Lehrfach, Kiel, Verein zur Förderung regionalwissenschaftlicher Analysen, 1975.

Becker, H. S., Geer, B., Hughes, E. C. , and Strauss, A.: Boys in white: student culture in medical school, Chicago, Chicago University Press, 1961.

Berndt, C.: Globalisierungs-Grenzen: Modernisierungsträume und Lebenswirklichkeiten in Nordmexiko, Bielefeld, transcript, 2004.

Boeckler, M. und Berndt, C.: Kulturelle Geographien der Ökonomie: zur Performativität von Märkten, in: Kulturelle Geographien: zur Beschäftigung mit Raum und Ort nach dem Cultural Turn, Herausgeber: Berndt, C. und Pütz, R., Bielefeld, 213-258, 2008.

Clifford, J. und Marcus, G. E. (Hrsg.): Writing culture: the poetics and politics of ethnography, Berkeley, University of California Press, 1986.

Crang, M. und Cook, I.: Doing ethnographies, London, Sage, 2007.

Curran, J.: Agatha Christie's secret notebooks: fifty years of mysteries in the making, New York, HarperCollins, 2009.

Denzin, N.: Interpretive ethnography: ethnographic practices for the 21st century, Thousand Oaks, CA, Sage, 1997.

Dirksmeier, P. and Helbrecht, I.: Intercultural interaction and "situational places": a perspective for urban cultural geography within and beyond the performative turn, Soc. Geogr., 5, 39-48, doi:10.5194/sg-5-39-2010, 2010.

Everts, J.: Konsum und Multikulturalität im Stadtteil, Bielefeld, transcript, 2008.

Everts, J., Lahr-Kurten, M., und Watson, M.: Practice matters! Geographical inquiry and theories of practice, Erdkunde, 65, 323 334, 2011

Falzon, M.-A. (Hrsg.): Multi-sited ethnography: theory, praxis and locality in contemporary research, London, Ashgate, 2009.

Fine, G. A.: Ten lies of ethnography: Moral dilemmas of field research, J. Contemp. Ethnogr., 22, 267-294, 1994.

Fine, G. A.: Morel tales: the culture of mushrooming, Cambridge, MA, Harvard University Press, 1998.

Füller, H. und Marquardt, N.: Gouvernementalität in der humangeographischen Diskursforschung, in: Handbuch Diskurs und Raum, Herausgeber: Glasze, G. und Mattissek, A.: Bielefeld, 83-106, 2009.

Geertz, C.: The interpretation of cultures, New York, Basic Books, 1973.

Geertz, C.: Dichte Beschreibung: Beiträge zum Verstehen kultureller Systeme, Frankfurt, Suhrkamp, 1983 [1973].

Geertz, C.: Works and lives: the anthropologist as author, Stanford, Stanford University Press, 1988.

Gille, Z. und Ó Riain, S.: Global ethnography, Annu. Rev. Sociol., 28, 271-295, 2002.

Hammersley, M. und Atkinson, P.: Ethnography: principles and practices, 2nd Edn., New York, Routledge, 1995.

Haraway, D.: Simians, cyborgs and women: the reinvention of nature, Routledge, London, 1991.

Herbert, S.: For ethnography, Prog. Hum. Geog. 24, 550-568, 2000.

Herrera, C. D.: A clash of methodology and ethics in "undercover" social sciences, Philos. Soc. Sci., 33, 351-362, 2003.

Humphreys, L.: Tearoom trade: A study of homosexual encounters in public places, London, Duckworth, 1970.
Katz, C.: All the world is staged: intellectuals and the project of ethnography, Environ. Plann. D, 10, 495-510, 1992.

Korf, B.: Die Ordnung der Entwicklung: zur Ethnographie der Entwicklungspraxis und ihrer ethischen Implikationen, Geogr. Z., 92, 208-226, 2004.

Lange, B.: Die Räume der Kreativszenen: culturepreneurs und ihre Orte in Berlin, Bielefeld, transcript, 2007.

Law, J.: After method, London, Routledge, 2004.

Madison, D. S.: Critical ethnography: method, ethics and performance, London, Sage, 2005.

Marcus, G. E.: What comes (just) after "post"? The case of ethnography, in: The handbook of qualitative research, Herausgeber: Denzin, N. und Lincoln, Y. S., London, 563-574, 1994.

Mauss, M.: Manuel d'ethnographie, Paris, Payot, 1947.

Megoran, N.: For ethnography in political geography: experiencing and re-imagining Ferghana Valley boundary closures, Polit. Geogr., 25, 622-640, 2006.

Müller, M.: Reconsidering the concept of discourse in the field of critical geopolitics: towards discourse as language and practice, Polit. Geogr., 27, 322-338, 2008.

Müller, M.: Making great power identities in Russia: an ethnographic discourse analysis of education at a Russian elite university, Zürich, LIT, 2009.

Orr, J.: Talking about machines: an ethnography of a modern job, Ithaca, Cornell University Press, 1996.

Peck, J. und Theodore, N.: Mobilizing policy: models, methods and mutations, Geoforum, 41, 169-174, 2010.

Rose, G.: Situating knowledges: positionality, reflexivities and other tactics, Prog. Hum. Geog., 21, 305-320, 1997.

Rothfuß, E.: Exklusion im Zentrum: die brasilianische Favela zwischen Stigmatisierung und Widerständigkeit, Bielefeld, transcript, 2012.

Sanjek, R. (Hrsg.): Fieldnotes: the makings of anthropology, Ithaca, Cornell University Press, 1990.

Schurr, C. und Segebart, D.: Challenging ways of doing development geography through feminist postcolonialism, Geogr. Helv., im Druck, 2013.

Strüver, A.: Der Konstruktivismus lernt laufen: „Doing morethan-representational geography“", Soc. Geogr., 6, 1-13, doi:10.5194/sg-6-1-2011, 2011.

Trinh, T. M.-H.: When the moon waxes red: representation, gender and cultural politics, New York, Routledge, 1991.

van Maanen, J.: Tales of the field: on writing ethnography, Chicago, University of Chicago Press, 1988.

Visweswaran, K.: Fictions of feminist ethnography, Minneapolis, University of Minnesota Press, 1994.

Wastl-Walter, D.: Gender Geographien: Geschlecht und Raum als soziale Konstruktionen, Stuttgart, Steiner, 2010.

Watson, A. und Till, K. E.: Ethnography and participant observation, in: The Sage Handbook of Qualitative Geography, Herausgeber: DeLyser, D., Herbert, S., Aitken, S., Crang, M., und McDowell, L., London, 121-137, 2010.

Whyte, W. F.: Street Corner Society: the social structure of an Italian slum, Chicago, University of Chicago Press, 1943.

Wolcott, H. F.: The man in the principle's office: an ethnography, New York, Holt, Rinehart and Winston, 1973.

Wolcott, H. F.: Ethnography: a way of seeing, Walnut Creek, Altamira Press, 1999. 